Check for updates

Cite this: RSC Adv., 2019, 9, 7086

Received 8th January 2019

Accepted 4th February 2019

DOI: 10.1039/c9ra00167k

rsc.li/rsc-advances

\title{
The mechanism and activity of oxygen reduction reaction on single atom doped graphene: a DFT method $\uparrow$
}

\author{
Xiaoming Zhang, ${ }^{a}$ Zhangxun Xia, ${ }^{a}$ Huanqiao Li, ${ }^{a}$ Shansheng $Y u,{ }^{1}{ }^{b}$ Suli Wang*a \\ and Gongquan Sun (D) *a
}

\begin{abstract}
Heteroatom doped graphene as a single-atom catalyst for oxygen reduction reaction (ORR) has received extensive attention in recent years. In this paper, the ORR activity of defective graphene anchoring single heteroatom (IIIA, IVA, VA, VIA and VIIA) was systematically investigated using a dispersion-corrected density functional theory method. For all of the 34 catalysts, 14 of which were further analyzed, and the Gibbs free energy of each elementary reaction was calculated. According to the scaling relationship between $\Delta G_{\mathrm{OOH}^{*}}$ and $\Delta G_{\mathrm{OH}^{*}}$, we further analyzed the rate-determining step of the remaining 20 catalysts. The results show that when the ORR reaction proceeds in the path $\mathrm{O}_{2} \rightarrow \mathrm{OOH} \rightarrow \mathrm{O} \rightarrow \mathrm{OH}$ $\rightarrow \mathrm{H}_{2} \mathrm{O}$, the reaction energy barriers are lower than $0.8 \mathrm{eV}$ for Te-SV, Sb-DV, Pb-SV, Pb-DV, As-SV, AsDV, B-SV, Sn-SV and N-SV. Our result provides a theoretical basis for further exploration of carbonbased single-atom catalysts for ORR.
\end{abstract}

\section{Introduction}

Proton exchange membrane fuel cells (PEMFCs) are one of the most promising clean energy technology candidates with simplicity, low working temperature, high power density, and quick start-up characteristics. These advantages promote their widespread commercial applications in the areas of transportation, stationary and portable power generation. However, to achieve a desirable fuel cell performance, the sluggish reaction kinetics of the oxygen reduction reaction (ORR) requires much higher Pt loading $\left(\sim 0.4 \mathrm{mg} \mathrm{cm}^{-2}\right)$, even on the best Ptbased catalyst. Due to the scarcity and high price of Pt metal, reducing the cathode Pt loading or even completely replacing it with abundant and cheap catalysts would be advantageous. In recent years, a large number of non-precious metal catalysts (NPMCs) have been explored to substitute Pt in ORR, such as metal oxide, metal carbide, metal nitride and carbon based catalysts. ${ }^{1-3}$ Among all of the NPMCs, graphene and carbon nanotube (CNT) based materials receive widespread attention due to their low price, fuel tolerance, good electrical and mechanical properties, high surface area, and noticeable electrochemical durability under actual working environments. ${ }^{4,5}$

${ }^{a}$ Division of Fuel Cells and Battery, Dalian National Laboratory for Clean Energy, Dalian Institute of Chemical Physics, Chinese Academy of Sciences, Dalian 116023, China.E-mail: gqsun@dicp.ac.cn; suliwang@dicp.ac.cn

${ }^{b}$ Department of Materials Science, Jilin University, Changchun 130012, China

$\dagger$ Electronic supplementary information (ESI) available. See DOI: $10.1039 / \mathrm{c} 9 \mathrm{ra} 00167 \mathrm{k}$
As an effective method, doping heteroatoms into a graphene matrix could adjust their electronic properties and enhance the ORR performance. Tremendous efforts have been undertaken to this filed, both on the experimental and theoretical level. ${ }^{6-11}$ Dai's group $^{6,12}$ is one of the pioneers in the study of nitrogen-doped carbon material for ORR. They synthesized nitrogen-doped CNT (N-CNT) and graphene (N-graphene) by pyrolyzing and chemical vapor deposition of methane in the presence of $\mathrm{NH}_{3}$. The resultant N-CNT and N-graphene were demonstrated to act as metal-free electrode with a much better electrocatalytic activity, long-term operation stability, and tolerance to crossover effect than platinum for oxygen reduction via a four-electron pathway in alkaline fuel cells. Sheng et al. ${ }^{13}$ prepared boron doped graphene (B-graphene) on a large scale using boron oxide as the boron source. Compared to pristine graphene, the Bgraphene exhibits excellent electrocatalytic activity and longterm stability towards ORR in alkaline electrolytes, and good tolerance to CO poisons. Hu et al. ${ }^{14}$ further explored the ORR activity of boron and nitrogen co-doped carbon nanotubes (BNCNTs). As for the two kinds of BN-CNTs, bonded or separated $\mathrm{B}$ and $\mathrm{N}$, it was found that only the separated one can turn the inert CNTs into ORR electrocatalysts from both experimental and theoretical results. This progress demonstrates the crucial role of the doping microstructure on ORR performance, which is significant for further exploring the advanced C-based metal-free electrocatalysts. The enhanced ORR activity of carbon-based materials through $\mathrm{B}$ and $\mathrm{N}$ doping further stimulates research interest in other doping systems. Yang and his collaborators ${ }^{15}$ reported sulfur/selenium-doped graphene through a simple and economical approach. They confirmed the potential use of S- 
graphene in direct methanol and alkaline fuel cells. Wei et al. ${ }^{16}$ successfully prepared phosphorus-doped graphene nanosheets and found that the $\mathrm{P}$ atoms were substitutional incorporated into the carbon framework and were partially oxidized, which created new active sites for ORR. Gong et al. ${ }^{17}$ fabricated nitrogen- and phosphorus-doped biocarbon via a simple and low-cost biosynthesis method using yeast cells as a precursor. The as-prepared biocarbon exhibits an onset potential of $-0.076 \mathrm{~V}(v s . \mathrm{Ag} / \mathrm{AgCl})$ and a negative shift of only about $29 \mathrm{mV}$ in the half-wave potential of the biocarbon compared to commercial Pt/C. In addition, other two-dimensional materials have also been widely used as catalysts in various reactions, such as water splitting, ${ }^{18}$ CO oxidation ${ }^{19}$ and ORR. ${ }^{20,21}$ However, the key ORR activity mechanisms of metal-free materials are still incomparable with those of Pt-based catalysts, which stimulate the on-going debate on active sites and reaction pathway for ORR. In view of the significant influence of raw materials, preparation methods, post-treatment processes on the structure and properties of carbon-based catalysts and the lack of in situ characterization techniques, more promising high activity ORR catalysts could be predicted by screening more heteroatom-doped carbon materials with density functional theory (DFT) method. Although Me-N-C has been a very promising ORR catalyst, it is not the focus of this article. $^{22-24}$

The research and design of single-atom catalysts (SACs) requires a combination of the geometric and electronic properties of the single atoms themselves, as well as the geometric and electronic properties of the substrate. We have systematically studied the $3 \mathrm{~d}$ metal atoms-B/C/N modified defective graphene ${ }^{25}$ and $\mathrm{A}(\mathrm{III}-\mathrm{VI})$ group atoms- $\mathrm{MoS}_{2}$ with in-plane $\mathrm{S}$ defect. ${ }^{26}$ In this work, we will systematically study the geometric and electronic properties of IIIA, IVA, VA and VIA monoatomic doped single and double vacancies graphene (short as X-SV and $\mathrm{X}-\mathrm{DV}$ ) catalysts. The stability and ORR activities of X-SV/DV have been further evaluated. Density functional theory (DFT) calculations are used to quantify the binding energies and reaction energetics of surface species involved in ORR.

\section{Method}

\subsection{The thermodynamics of the ORR}

The ORR activities on active sites of heteroatom-doped defective graphene were studied in detail. In alkaline environment, the oxygen molecular could be reduction to $\mathrm{OH}^{-}$, and the detail reaction scheme is shown as below:

$$
\begin{gathered}
\mathrm{O}_{2}(\mathrm{~g})+\mathrm{H}_{2} \mathrm{O}(\mathrm{l})+\mathrm{e}^{-}+* \rightarrow \mathrm{OOH}^{*}+\mathrm{OH}^{-} \\
\mathrm{OOH}^{*}+\mathrm{e}^{-} \rightarrow \mathrm{O}^{*}+\mathrm{OH}^{-} \\
\mathrm{O}^{*}+\mathrm{H}_{2} \mathrm{O}(\mathrm{l})+\mathrm{e}^{-} \rightarrow \mathrm{OH}^{*}+\mathrm{OH}^{-} \\
\mathrm{OH}^{*}+\mathrm{e}^{-} \rightarrow *+\mathrm{OH}^{-}
\end{gathered}
$$

where * refers to a possible active site in X-SV/DV model. (l) and (g) refer to the liquid and gas phases, respectively. ${ }^{*} \mathrm{O},{ }^{*} \mathrm{OH}$ and * $\mathrm{OOH}$ are the adsorbed intermediates.
For each step, the reaction free energy $\Delta G$ is defined as the difference between free energies of the initial and final states and is given by the expression, ${ }^{27}$

$$
\begin{gathered}
\Delta G_{1}=\Delta G_{\mathrm{OOH}^{*}}-4.92 \mathrm{eV}+\Delta G(\mathrm{pH})+e U \\
\Delta G_{2}=\Delta G_{\mathrm{O}^{*}}-\Delta G_{\mathrm{OOH}^{*}}+\Delta G(\mathrm{pH})+e U \\
\Delta G_{3}=\Delta G_{\mathrm{OH}^{*}}-\Delta G_{\mathrm{O}^{*}}+\Delta G(\mathrm{pH})+e U \\
\Delta G_{4}=-\Delta G_{\mathrm{OH}^{*}}+\Delta G(\mathrm{pH})+e U
\end{gathered}
$$

The Gibbs free energies of eqn (5)-(8) $(\Delta G)$ are related to the adsorption energies of the various intermediate species. The adsorption free energy changes of these intermediate species are determined using ${ }^{28}{ }^{28} \Delta G_{\mathrm{ads}}=\Delta E_{\mathrm{ads}}^{\mathrm{DFT}}+\Delta \mathrm{ZPE}-T \Delta S$, where $\Delta E_{\text {ads }}^{\text {DFT }}$ can be calculated relative to $\mathrm{H}_{2} \mathrm{O}$ and $\mathrm{H}_{2}$. The absorption energies were calculated as follows, $\Delta E_{\mathrm{OH}^{*}}=E\left(\mathrm{OH}^{*}\right)-E\left({ }^{*}\right)-$ $\left(E_{\mathrm{H}_{2} \mathrm{O}}-1 / 2 E_{\mathrm{H}_{2}}\right), \Delta E_{\mathrm{OOH}^{*}}=E\left(\mathrm{OOH}^{*}\right)-E\left({ }^{*}\right)-\left(2 E_{\mathrm{H}_{2} \mathrm{O}}-3 / 2 E_{\mathrm{H}_{2}}\right)$, $\Delta E_{\mathrm{O}^{*}}=E\left(\mathrm{O}^{*}\right)-E\left({ }^{*}\right)-\left(E_{\mathrm{H}_{2} \mathrm{O}}-E_{\mathrm{H}_{2}}\right), E_{\mathrm{H}_{2} \mathrm{O}}$ and $E_{\mathrm{H}_{2}}$ are the calculated DFT energies of $\mathrm{H}_{2} \mathrm{O}$ and $\mathrm{H}_{2}$ molecules in the gas phase using the approaches outlined by Nørskov et al. ${ }^{28,29} \Delta \mathrm{ZPE}$ and $T \Delta S$ are the zero point energy difference and the entropy change between the absorbed state and the free state, i.e., the gas phase, respectively, and $T$ is the temperature $(298.15 \mathrm{~K}$ in this work). $e$ is the elementary charge and $U$ is the potential difference between the electrode and the normal hydrogen electrode (NHE). The free energy change of $\mathrm{H}^{+}$is derived according to $\Delta G_{\mathrm{pH}}=k_{\mathrm{B}} T \ln (10) \times \mathrm{pH},\left(k_{\mathrm{B}}\right.$ is Boltzmann's constant, and $\mathrm{pH}=13$ for alkaline medium). Because the highspin ground state of an oxygen molecule is difficult to describe in DFT calculations, the free energy of $\mathrm{O}_{2}(\mathrm{~g})$ is derived as $G_{\mathrm{O}_{2}}(\mathrm{~g})$ $=2 G_{\mathrm{H}_{2} \mathrm{O}}(\mathrm{l})-2 G_{\mathrm{H}_{2}}(\mathrm{~g})+4.92 \mathrm{eV}$.

\subsection{Computational details}

The spin-polarized DFT calculations were performed by the $\mathrm{DMol}^{3}$ code embedded in Materials Studio ${ }^{30}$ with long-range dispersion correction via Grimme's scheme. ${ }^{31}$ The generalized gradient approximation with the Perdew-Burke-Ernzerhof $(\mathrm{PBE})^{32}$ functional is employed to describe exchange and correlation effects. The all electrons core treat method is implemented. The double numerical atomic orbital augmented by a polarization function is chosen as the basis set. ${ }^{33}$ During geometrical optimization, the basis set cut-off was chosen to be $5.2 \AA$. The convergence tolerances for the geometry optimization were set to $10^{-5} \mathrm{Ha}$ for the energy, $0.002 \mathrm{Ha} \AA^{-1}$ for the force, and $0.005 \AA$ for the displacement. The electronic SCF tolerance was set to $10^{-6}$ Ha. A Fermi smearing parameter of $0.005 \mathrm{Ha}$ was used in the calculations. The reciprocal space was sampled with a $6 \times 6 \times 1 k$-points grid generated automatically using the Monkhorst-Pack method ${ }^{34}$ for the relaxation calculations and a $(18 \times 18 \times 1) k$-points grid was used for electronic structure computations. The conductor-like screening model $(\mathrm{COSMO})^{35}$ is used to simulate the aqueous environment, where the dielectric constant is set as 78.54 (water). First-principles molecular dynamics (MD) was performed with NVE method. A time step of $1 \mathrm{fs}$ was used and the temperature was set to 353.15 
K. We have conducted a convergence test for important parameters (orbital cut-off quality and $k$-point set) and slab size, and the results indicate that present parameters and $p(4 \times 4)$ slab are fine enough. More detail information is shown in Table S1. $\dagger$ Our DFT calculated lattice parameter for graphite is $2.47 \AA$ which is consistent with the experimental value and other computational results. ${ }^{36}$ More than $15 \AA$ thick vacuum is added to avoid the artificial interactions between graphene and its images. In all of the structure optimization calculations, all the atoms are fully relaxed.

\section{Results and discussions}

\subsection{The geometric and electronic properties}

The geometry-optimized graphene sheet has an in-plane $\mathrm{C}-\mathrm{C}$ distance of $1.42 \AA$, which is consistent with the previous results. ${ }^{25,36}$ The single and double vacancies of graphene were constructed through removing one and two neighbouring atoms, respectively. When a single hetero-atom is introduced into the defect position in graphene, the $\mathrm{C}$ atoms with dangling bonds at the defect will capture and anchor the hetero-atom. The geometric and electronic properties of $\mathrm{X}-\mathrm{SV} / \mathrm{DV}(\mathrm{X}=\mathrm{B}$, C, N, O; Al, Si, P, S; Ga, Ge, As, Se; In, Sn, Sb, Te; Tl, Pb, Bi) catalysts have been analysed in details.

3.1.1 Geometric properties. Depending on the size of the hetero-atoms and defect, the X-SV have two different structures, one being a planar structure, as shown in Fig. 1(a), including B, $\mathrm{C}, \mathrm{N}, \mathrm{O}$. The atomic radius of $\mathrm{B}(0.88), \mathrm{N}(0.70)$ and $\mathrm{O}(0.73)$ are close to the $\mathrm{C}(0.77)$, so the planar structure can be maintained after replacing the $\mathrm{C}$ atom position. According to Table $\mathrm{S} 2, \uparrow$ the impurity atoms (B, N, O) are kept in the same plane as the graphene, and the height difference is zero. Second, the convex structure, as shown in Fig. 1(b), includes Al, Si, P, S; Ga, Ge, As, $\mathrm{Se}$; $\mathrm{In}, \mathrm{Sn}, \mathrm{Sb}, \mathrm{Te} ; \mathrm{Tl}, \mathrm{Pb}$ and $\mathrm{Bi}$. For a dopant atom with a large atomic radius, such as $\mathrm{Al}$ (1.43), $\mathrm{Si}$ (1.17), Ga (1.35), and In (1.67) etc., the single atom vacancy defect is not sufficient to accommodate such a large impurity atom, so the heteroatom will protrude from the graphene plane and form a convex structure. The difference in height between the impurity atoms and the plane of the graphene is related to the radius of the impurity atoms. For most systems, the larger the atomic radius, the longer the $\mathrm{X}-\mathrm{C}$ bond length as well as the greater the height of the $\mathrm{X}$ atoms, as shown in Table S2.† For X-DV systems, the situation is similar. It was found that some X-DV systems have

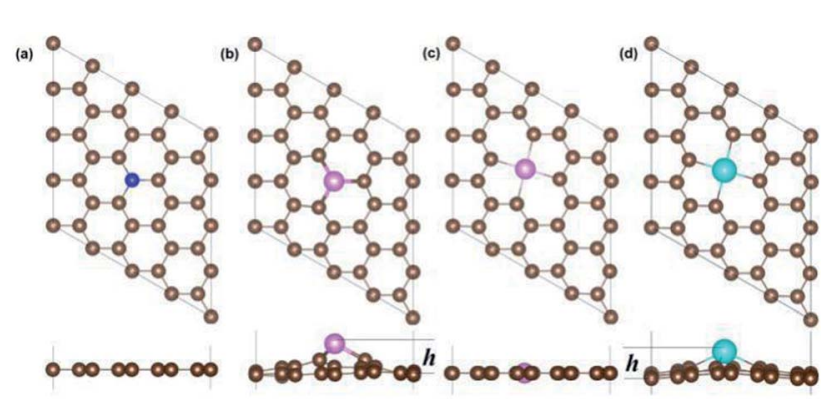

Fig. 1 The optimized geometry structure of N-SV (a), Al-SV(b), Al-DV (c) and In-DV (d). a planar structure (X = Al, Si, P, S, Ga, Ge, As) and other X-DVs have a non-planar structure. The reason is similar to the X-SV system. See Table $\mathrm{S} 3 \uparrow$ for specific data.

3.1.2 Electronic properties. In addition, we studied the density of states and Mulliken charge of the X-SV/DV system. It is found that, except for the $\mathrm{N \& O}$ doping system, the $\mathrm{X}$ atoms are positively charged, and the adjacent $\mathrm{C}$ atoms coordinating with $\mathrm{X}$ are negatively charged, as shown in Tables S2 and S3. $\dagger$ The greater the difference in electronegativity between the $\mathrm{X}$ atom and the $\mathrm{C}$ atom, the more pronounced the charge transfer between the two. The density of states analysis of the $\mathrm{X}$ atom reveals that the average energy (the sp electrons band center) changes regularly. For the $\mathrm{X}$ atom of the same period, the center of the sp band satisfies $\mathrm{B}>\mathrm{C}>\mathrm{N}>\mathrm{O}$, as shown in Table $\mathrm{S} 2 . \dagger$ The trend of $\mathrm{X}-\mathrm{DV}$ is similar to $\mathrm{X}-\mathrm{SV}$.

\subsection{The stability}

Stability is an important parameter for judging catalyst performance. To evaluate the stability of the catalyst, the bonding energy between the $\mathrm{X}$ atom and the defective graphene in the $\mathrm{X}$ SV/DV catalyst was defined as following:

$$
\Delta E_{\mathrm{b}}=E_{\mathrm{X}-\mathrm{SV} / \mathrm{DV}}-E_{\mathrm{SV} / \mathrm{DV}}-E_{\mathrm{X}}
$$

where $E_{\mathrm{X}-\mathrm{SV} / \mathrm{DV}}, E_{\mathrm{SV} / \mathrm{DV}}$ and $E_{\mathrm{X}}$ are the total energies of the single atom doped graphene systems, defective graphene and an isolated $\mathrm{X}$ atoms, respectively. It was found that the X-SV bonding energy for most systems is greater than the corresponding X-DV. The stability of the catalyst is closely related to the size and electronic structure of the dopant atoms. We found that for small atomic radius doping SV systems, the stability decrease in the order: $\mathrm{C}(\mathrm{p} 2)>\mathrm{B}(\mathrm{p} 1)>\mathrm{N}(\mathrm{p} 3)>\mathrm{O}(\mathrm{p} 4)$, with regard to large atomic radius doping $\mathrm{SV}$ system, the change trends are somewhat different (as shown in Table $\mathrm{S} 4 \dagger): \mathrm{P}(\mathrm{p} 3)>\mathrm{Si}(\mathrm{p} 2)>\mathrm{S}(\mathrm{p} 4)>$ $\mathrm{Al}(\mathrm{p} 1)[\mathrm{As}(\mathrm{p} 3)>\mathrm{Ge}(\mathrm{p} 2)>\mathrm{Se}(\mathrm{p} 4)>\mathrm{Ga}(\mathrm{p} 1)],[\mathrm{Sb}(\mathrm{p} 3)>\mathrm{Sn}(\mathrm{p} 2)>$ $\mathrm{Te}(\mathrm{p} 4)>\operatorname{In}(\mathrm{p} 1)],[\mathrm{Bi}(\mathrm{p} 3)>\mathrm{Pb}(\mathrm{p} 2)>\mathrm{Tl}(\mathrm{p} 1)]$. For large-sized doped DV systems: $\mathrm{Si}(\mathrm{p} 2)>\mathrm{P}(\mathrm{p} 3)>\mathrm{S}(\mathrm{p} 4)>\mathrm{Al}(\mathrm{p} 1)[\mathrm{As}(\mathrm{p} 3)>\mathrm{Ge}(\mathrm{p} 2)>$ $\mathrm{Se}(\mathrm{p} 4)>\mathrm{Ga}(\mathrm{p} 1)],[\mathrm{Sb}(\mathrm{p} 3)>\mathrm{Te}(\mathrm{p} 4)>\mathrm{Sn}(\mathrm{p} 2)>\operatorname{In}(\mathrm{p} 1)],[\mathrm{Tl}(\mathrm{p} 1)>$ $\mathrm{Bi}(\mathrm{p} 3)>\mathrm{Pb}(\mathrm{p} 2)]$, the regularity of the system is poor, and the atomic radius is also used. The electronic structure is explained: the density of states (DOS) calculation shows that there is a clear orbital overlap between the $\mathrm{X}$ atom and the $\mathrm{C}$ atom, and there is a covalent bond between the two. The calculation of differential charge density and Mulliken charge shows that there is a significant charge transfer between $\mathrm{X}$ and $\mathrm{C}$, and there is a significant ionic bond between the two. Therefore, the $\mathrm{X}-\mathrm{C}$ forms a hybrid bond. There is no significant spin change in the material, so it can be considered as a zero spin material. After introducing the aqueous environment effect, the stability of the catalyst is almost unchanged, as shown in Table S4. $\dagger$ Furthermore, first-principles molecular dynamics in a period of $1000 \mathrm{fs}$ (1 ps) calculations at the temperature of $353.15 \mathrm{~K}$ were conducted for Tl-SV $\left(\Delta E_{\mathrm{b}}=-0.23 \mathrm{eV}\right)$, In-SV $\left(\Delta E_{\mathrm{b}}=-2.66 \mathrm{eV}\right)$ and Ga-SV $\left(\Delta E_{\mathrm{b}}=-4.35 \mathrm{eV}\right)$. It was found that only $\mathrm{Ga}$ and $\mathrm{C}$ atoms in Ga-SV can maintain bonding with slight distortion, and the structures of other systems were destroyed, as shown in Fig. S1. $\dagger$ This indicates that only catalysts with a bonding energy 
greater than $4 \mathrm{eV}$ could remain stable under PEMFC operating conditions.

The binding properties of $\mathrm{X}$ atom on vacancy are analysed by calculating the charge density difference in real space. The charge density difference of $\mathrm{X}-\mathrm{V}$ is defined as following equation and shown in Fig. 3:

$$
\Delta \rho=\rho_{\mathrm{X}-\mathrm{V}}-\rho_{\mathrm{V}}-\rho_{\mathrm{X}}
$$

where the charge density is calculated in the same supercell for all quantities, $\rho_{\mathrm{X}-\mathrm{V}}$ is the total charge density of the X-vacancy system, and $\rho_{\mathrm{V}}$ and $\rho_{\mathrm{X}}$ are the charge densities of noninteracting pristine vacancy and $\mathrm{X}$ atom, respectively. The charge density difference quantifies the redistribution of electron charge due to the interaction between adatom and vacancy. The accumulated charge density around the $\mathrm{X}-\mathrm{C}$ bonds indicates that $\mathrm{X}$ is strongly bound on the site vacancy of graphene, in accordance with the high exothermic adsorption energy in Fig. 2 and Table $\mathrm{S} 4, \dagger$ implying that the covalent bonding is formed between $\mathrm{X}$ and $\mathrm{C}$ atoms. In addition, Fig. 3 shows a charge transfer from $\mathrm{X}$ to $\mathrm{C}$ atom, which is consistent with the electronegativity difference between $\mathrm{C}$ and $\mathrm{X}$ atoms. The Mulliken charge of the $\mathrm{X}$-vacancy systems is calculated in Table $\mathrm{S} 3, \dagger$ which also indicates that a great amount of electrons are transferred from $\mathrm{X}$ to substrate. The amount of charge transformed from $\mathrm{X}$ to $\mathrm{C}$ decrease in the order $\mathrm{Al}>\mathrm{Si}>\mathrm{P}>\mathrm{S}$, as observed in Fig. 3 and Table $\mathrm{S} 2 \uparrow$ for both $\mathrm{X}$-SV and X-DV. Also, the X-DV systems are more significant due to more $\mathrm{X}-\mathrm{C}$ bonds. This phenomenon indicates the obvious ionic bond characteristic.

What's more, there exist significant electronic density overlap between $X$ and substrate, as shown in Fig. 4. This phenomenon indicates the obvious covalent bond feature. The corresponding orbits at specific energy levels have also been given in the inset pictures in Fig. 4.

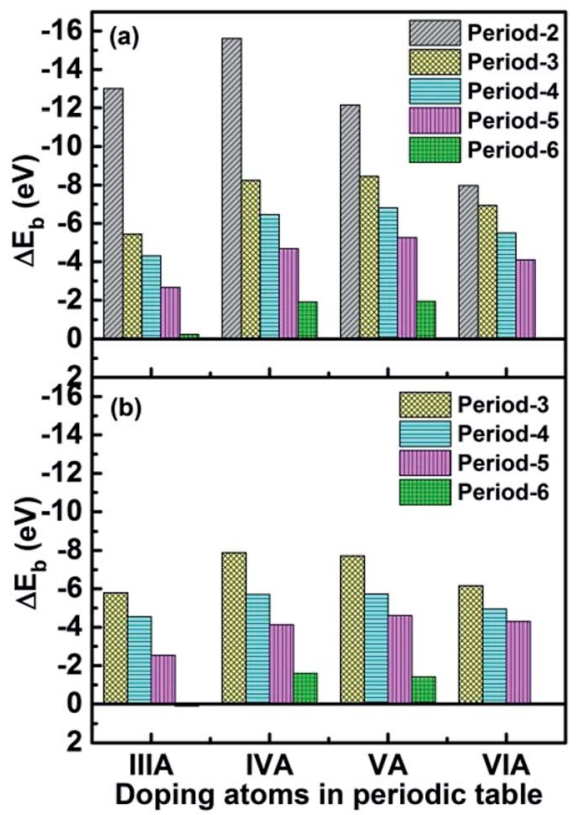

Fig. 2 The binding energies between $X$ atoms and defective graphene for X-SV (a) and X-DV (b).

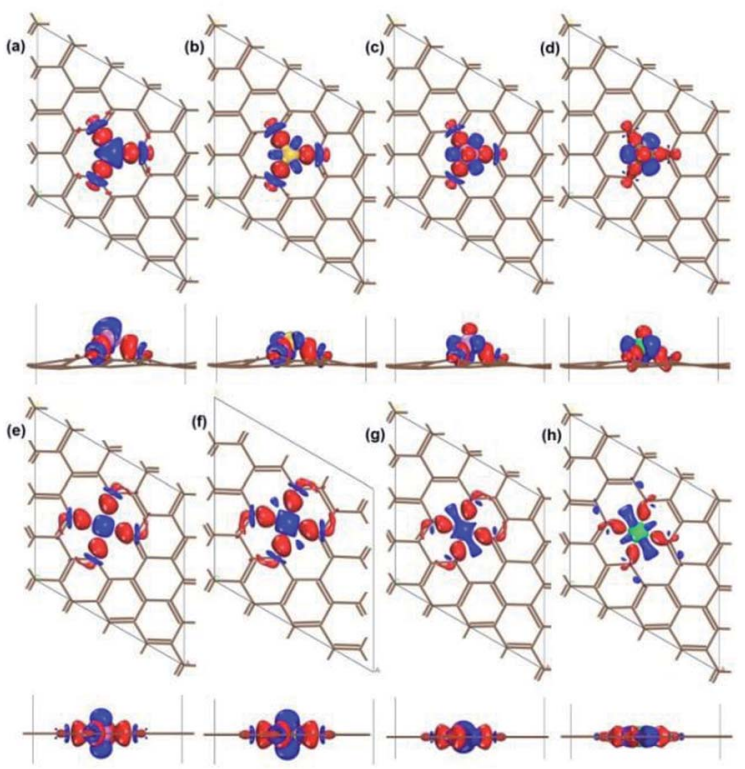

Fig. 3 Top view and side view of the charge density difference for Al/ $\mathrm{Si} / \mathrm{P} / \mathrm{S}$ atom adsorbed on vacancy complexes: Al-SV (a), Si-SV (b), P-SV (c), S-DV (d), Al-DV (e), Si-DV (f), P-DV (g); S-DV (h). The isosurface value is 0.06 e $\AA^{-3}$. The red colour represents electron accumulation, while the blue colour represents electron depletion. The intensity of colour depends on the amount of electron change: the darkest red marks the most accumulation; the darkest blue marks the most depletion. Brown, C atom; purple, Al atom; yellow, Si atom; pink, P atom; green, $\mathrm{S}$ atom.

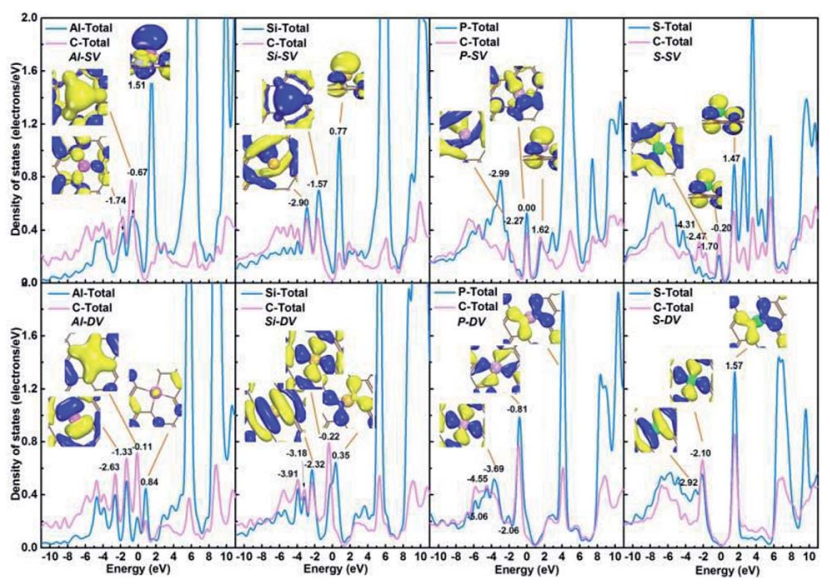

Fig. 4 PDOS of Al-SV, Si-SV, P-SV, S-SV, Al-DV, Si-DV, P-DV; S-DV. The corresponding orbits at specific energy levels are shown in the inset pictures.

\subsection{The ORR activities of X-SV/X-DV}

We selected 14 catalysts (according to the adsorption energy of $\mathrm{O}_{2}$ in the range of $\left.(0.3 \mathrm{eV}, 1.0 \mathrm{eV})\right)$ and analyzed the adsorption behavior of reactants $\left(\mathrm{O}_{2}\right)$, intermediates $\left(\mathrm{O}^{*}, \mathrm{OH}^{*}\right.$ and $\left.\mathrm{OOH}^{*}\right)$ and products $\left(\mathrm{H}_{2} \mathrm{O}\right)$ involved in the ORR reaction on the impurity atoms site. The optimized structures of oxygenated species on Al-SV and Al-DV have been shown in Fig. 5. The results show that the adsorption structure of oxygen-containing species is different for different substrates of the same impurity 


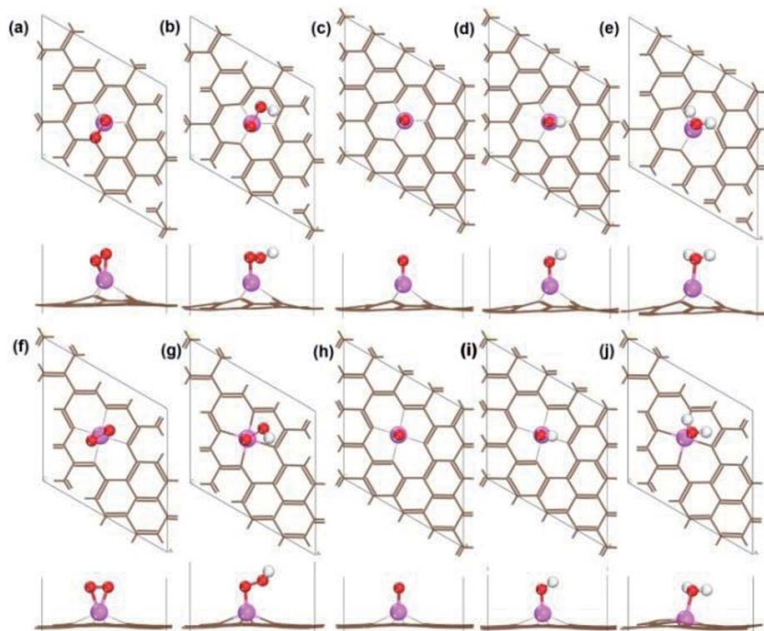

Fig. 5 The optimized geometry structure of $\mathrm{O}_{2}$ adsorbed Al-SV (a), $\mathrm{OOH}$ adsorbed Al-SV (b), O adsorbed Al-SV (c), OH adsorbed Al-SV (d), $\mathrm{H}_{2} \mathrm{O}$ adsorbed Al-SV (e), $\mathrm{O}_{2}$ adsorbed Al-DV (f), OOH adsorbed Al-DV (g), O adsorbed Al-DV (h), OH adsorbed Al-DV (i), $\mathrm{H}_{2} \mathrm{O}$ adsorbed Al-DV (j).

atom or different impurity atoms of the same substrate. Fig. 6(a) shows the adsorption free energies of $\mathrm{OOH}^{*}$ and $\mathrm{OH}^{*}$ for parts of X-SV and X-DV. The free energies of $\mathrm{OOH}^{*}$ are linearly related to that of $\mathrm{OH}^{*}$ by $y=0.91 x+3.40$. Fig. 6(b) shows the adsorption free energies of $\mathrm{OH}^{*}$ and $\mathrm{O}^{*}$ for parts of X-SV and X-DV structures. There is also a scaling relation with a slope of 1.04 between $\mathrm{OH}^{*}$ and $\mathrm{O}^{*}$ except some sites where oxygen atom is adsorbed in Al-DV, Ga-SV and so on. The free energy of reaction (1)-(4) can be calculated using eqn (5)-(8). According to the relationship between $\Delta G_{\mathrm{OH}^{*}}$ and $\Delta G_{\mathrm{O}^{*}}$, the catalysts are divided into two parts, and the detailed reaction free energy for each elemental steps were shown in Fig. 7(a) (linear relationship for

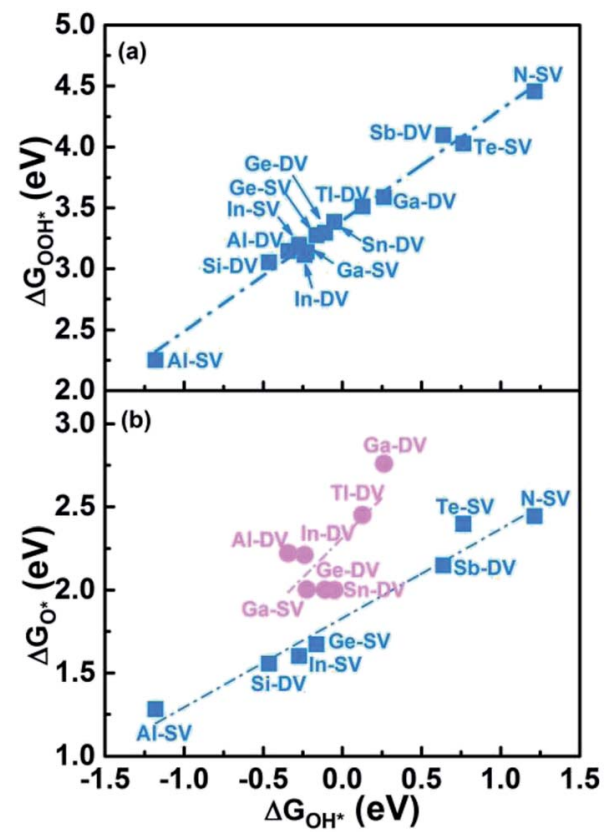

Fig. 6 The scaling relationship between $\Delta G_{\mathrm{OH}^{*}}$ and $\Delta G_{\mathrm{OOH}^{*}}$ (a) as well as $\Delta G_{\mathrm{OH}^{*}}$ and $\Delta G_{\mathrm{O} *}$ (b).

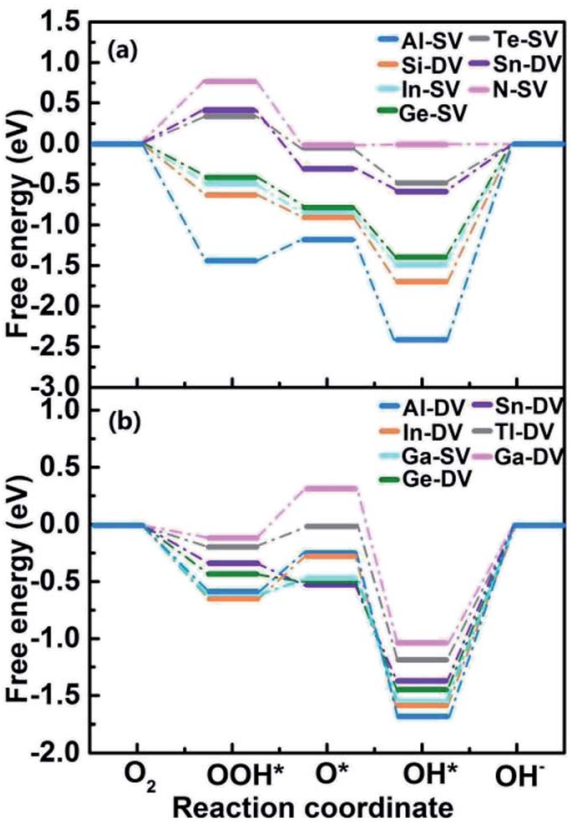

Fig. 7 The schematic Gibbs free energy diagrams of ORR for X-SV/DV with (a) or without (b) liner relationship.

Al-SV et al.) and Fig. 7(b) (nonlinear association for Al-DV et al.). For Al-SV et al. catalysts, it is found that the ORR either limited by step $1\left(\mathrm{O}_{2} \rightarrow \mathrm{OOH}^{*}\right)$ or step $4\left(\mathrm{OH}^{*} \rightarrow \mathrm{OH}^{-}\right)$, due to the weak or strong binding energy between oxygenated species and $\mathrm{X}$ graphene, respectively. As for step 1 , the energy barriers increase in the order Al-SV $<$ Si-DV $<$ In-SV $<$ Ge-SV $<$ Te-SV $<$ Sb$\mathrm{DV}<\mathrm{N}$-SV. An opposite trend is observed in step 4, that is Al-SV $>$ Si-DV $>$ In-SV $>$ Ge-SV $>$ Te-SV $>$ Sn-DV $>$ N-SV. For the overall reaction, the rate-determining step (RDS) of Te-SV $(0.76 \mathrm{eV}), \mathrm{Sb}$ $\mathrm{DV}(0.41 \mathrm{eV})$ and $\mathrm{N}-\mathrm{SV}(0.34 \mathrm{eV})$ are $\mathrm{O}_{2} \rightarrow \mathrm{OOH}^{*}$, and the RDS of Al-SV (2.41 eV), Si-DV (1.69 eV), In-SV (1.50 eV) and Ge-SV (1.39 $\mathrm{eV})$ are $\mathrm{OH}^{*} \rightarrow \mathrm{OH}^{-}$. For Al-DV et al. catalysts, the RDS of all seven catalysts are $\mathrm{OH}^{*} \rightarrow \mathrm{OH}^{-}$. The energy barriers increase in the order Ga-DV $(0.97 \mathrm{eV})<$ Tl-DV $(1.10 \mathrm{eV})<$ Sn-DV $(1.28 \mathrm{eV})<$ Ge-DV $(1.34 \mathrm{eV})<\mathrm{Ga}-\mathrm{SV}(1.46 \mathrm{eV})<\mathrm{In}-\mathrm{DV}(1.47 \mathrm{eV})<\mathrm{Al}-\mathrm{DV}(1.57$ $\mathrm{eV})$.

We further analyzed the RDS of the remaining 20 catalysts. First of all, we calculated the $\Delta G_{\mathrm{OH}^{*}}$ of all 20 catalysts. According to the scaling relationship between $\Delta G_{\mathrm{OOH}^{*}}$ and $\Delta G_{\mathrm{OH}^{*}}\left(\Delta G_{\mathrm{OOH}^{*}}\right.$ $=0.91 \Delta G_{\mathrm{O}^{*}}+3.40$ ), the $\Delta G_{\mathrm{OOH}^{*}}$ were then obtained (as shown in Table S7 $\dagger$ ). From the above, we can conclude that the RDS is either step 1 (determined by $\Delta G_{1}=\Delta G_{\mathrm{OOH}^{*}}-4.92 \mathrm{eV}+\Delta G(\mathrm{pH})$ $+e U$ ) or step 4 (determined by $\Delta G_{4}=-\Delta G_{\mathrm{OH}^{*}}+\Delta G(\mathrm{pH})+e U$ ). When $\mathrm{pH}=13, U^{\mathrm{NHE}}=0.44 \mathrm{~V}$, we could obtained $\Delta G_{1}$ and $\Delta G_{4}$ based on $\Delta G_{\mathrm{OH}^{*}}$. We compare the values of $\Delta G_{1}$ and $\Delta G_{4}$, and the maximum value was used as the energy barrier of RDS. Then, The relationship between $\Delta G_{\mathrm{OH}^{*}}$ and max energy barrier was summarized and shown in Fig. 8, Tables S8 and S9. $\dagger$ We found a "volcanic curve" relationship between $\Delta G_{\mathrm{OH}^{*}}$ and energy barrier. When the binding energy was too strong between $\mathrm{OH}^{*}$ and $\mathrm{SAC}\left(\Delta G_{\mathrm{OH}^{*}}<0.76 \mathrm{eV}\right)$, the oxygen reduction reaction was limited by $\mathrm{OH}^{*} \rightarrow \mathrm{OH}^{-}$, and the energy barrier decrease with $\Delta G_{\mathrm{OH}^{*}}$. As shown in Fig. 8, the identified catalyst 


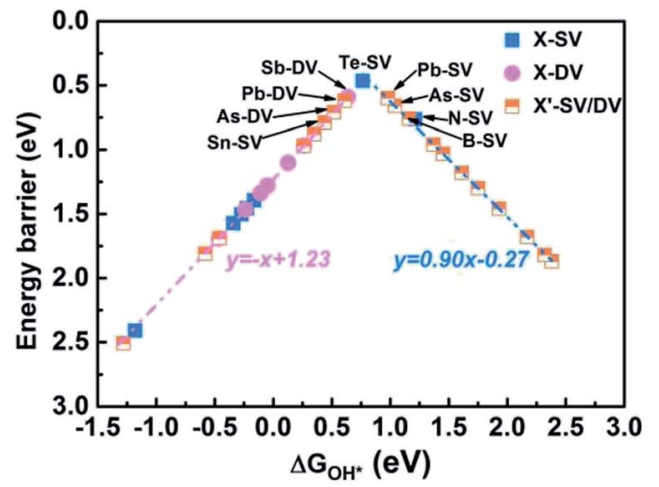

Fig. 8 The "volcanic curve" relationship between $\Delta G_{\mathrm{OH}^{*}}$ and energy barrier for all 34 catalysts.

energy barriers are lower than $0.8 \mathrm{eV}$ with $\mathrm{pH}=13$ and $U^{\mathrm{NHE}}=$ 0.44 . For the left side ones, the energy barrier increase in the order: Te-SV $(0.47 \mathrm{eV})<\mathrm{Sb}-\mathrm{DV}(0.59 \mathrm{eV})<\mathrm{Pb}-\mathrm{DV}(0.62 \mathrm{eV})<$ AsDV $(0.71 \mathrm{eV})<\mathrm{Sn}-\mathrm{SV}(0.79 \mathrm{eV})$, consistent with their increasing $\Delta G_{\mathrm{OH}^{*}}$ values. Further analysis of the difference between $\Delta G_{1}$ and $\Delta G_{1}\left(\Delta \Delta G=\Delta G_{4}-\Delta G_{1}\right)$, the same trend was found: Te-SV $(0.13 \mathrm{eV})<$ Sb-DV $(0.18 \mathrm{eV})<$ Pb-DV $(0.36 \mathrm{eV})<$ As-DV $(0.53 \mathrm{eV})<$ Sn-SV $(0.68 \mathrm{eV})$. This indicates that, the closer to the vertex of "volcanic curve", the closer the values between $\Delta G_{1}$ and $\Delta G_{4}$, and the smaller the energy barrier difference between the two elementary reactions. However, the farther the catalysts from the vertex of "volcanic curve", the bigger the gap between RDS and other steps. When the binding energy was too weak between $\mathrm{OH}^{*}$ and $\mathrm{SAC}\left(\Delta G_{\mathrm{OH}^{*}}>0.76 \mathrm{eV}\right)$, the oxygen reduction reaction was limited by $\mathrm{O}_{2} \rightarrow \mathrm{OOH}^{*}$, and the energy barrier decrease with $\Delta G_{\mathrm{OH}^{*}}$. The trend in right side is opposite to the left one. An ideal catalyst should be able to facilitate oxygen reduction just above the equilibrium potential, but requires all the four electrons transfer steps to have reaction free energies of the same magnitude at zero potential (i.e., $4.92 \mathrm{eV} / 4=1.23 \mathrm{eV}$ ). This is equivalent to all the reaction free energies being zero at the equilibrium potential, $1.23 \mathrm{~V}$ for acid medium and $0.44 \mathrm{~V}$ for alkaline medium with $\mathrm{pH}=13$. The reaction free energy $\Delta G_{1}$, $\Delta G_{2}, \Delta G_{3}$ and $\Delta G_{4}$ have the following relationship regardless of $\mathrm{pH}$ and potential correction:

$$
\begin{gathered}
\Delta G_{1}+\Delta G_{2}=\Delta G_{\mathrm{O}^{*}}-4.92 \mathrm{eV} \\
\Delta G_{3}+\Delta G_{4}=-\Delta G_{\mathrm{O}^{*}}
\end{gathered}
$$

Eqn (11) and (12) are competing with each other due to the opposite sign. Therefore, when $\Delta G_{1}, \Delta G_{2}, \Delta G_{3}$ and $\Delta G_{4}$ are equal, the RDS obtains the minimum value. As we have mentioned, when $\Delta \Delta G$ is the smallest, the lowest energy barrier obtained.

We also calculated the adsorption free energy and reaction free energy for all catalyst with aqueous environment. Although there are some differences in the absolute value of free energy, the general trend is almost same. Therefore, we put all related results in ESI (Fig. S2-S4†) for comparison and will not discuss here.

We also compared our results with other experimental and DFT results. Zhang et al. ${ }^{37}$ conducted research on four different
S-doped graphene quantum dot structures using the DFT method. Their calculations indicates that carbon atoms located at the zigzag edges or close to the $\mathrm{SO}_{2}$ doping structure possess high positive charge density or spin density, and the reaction can be carried out simultaneously through the four-electron path and the two-electron path on these carbon atoms. According to our calculations, however, both S-SV $(0.97 \mathrm{eV})$ and $\mathrm{S}-\mathrm{DV}(1.49 \mathrm{eV})$ have higher energy barriers at $0.9 \mathrm{~V}$, and the ratedetermining step is the same, both of which are $\mathrm{O}_{2}(\mathrm{~g})$ hydrogenation to form $\mathrm{OOH}^{*}$. We also compared the ORR activity of $\mathrm{N}$-SV catalyst with the results of Li et al. ${ }^{38}$ It was found that when consider the same reaction path, the rate-limiting step was same $\left(\mathrm{O}_{2}(\mathrm{~g})+\left(\mathrm{H}^{+}+\mathrm{e}^{-}\right)+* \rightarrow \mathrm{OOH}^{*}\right)$. However, our obtained overpotential values $(0.78 \mathrm{~V})$ was much higher than the ref. 38 . The differences in doping structure lead to differences in activity. The comparison of phosphorus doped graphene between our work and the others have also been done. ${ }^{39,40}$ The results obtained by Kaukonen et al. $^{39}$ indicate that single $\mathrm{P}$ atoms embedded into divacancies in graphene can be good candidates for ORR catalysts, which is consistent with our calculation results $(0.55 \mathrm{eV}$ at $0.9 \mathrm{~V}$ for P-DV). The DFT results of Zhang et $a .^{40}$ (P-SV) show that the reduction step from the second $\mathrm{OH}$ to $\mathrm{H}_{2} \mathrm{O}$ is the rate-determining step, which has a larger barrier of $0.88 \mathrm{eV}$, which is also consistent with our results, the same rate-limiting step and high energy barrier $(1.36 \mathrm{eV})$ at $0.9 \mathrm{~V}$. The difference in specific values is duo to the different method of evaluating barriers. We even compared with platinum-based ORR catalysts and found that the most active Te-SV has a reaction barrier comparable to $\mathrm{Pt}(211){ }^{41,42}$ In this article, we did not consider all possible active sites in detail, such as not considering carbon atoms adjacent to heteroatom. Therefore, the conclusions we give in this paper are a preliminary judgment. Next, we will specifically study the catalyst with high activity in a more detailed manner. By further analysing the various reaction pathways and all possible active sites, our ultimate goal is to finally confirm the high activity characteristics of the catalysts and achieve laboratory preparation of highly active catalysts.

\section{Conclusions}

In summary, we have systematically investigated the geometric property, electronic property, stability and ORR activity of 34 SACs based on defective graphene (short as X-SV or X-DV, $\mathrm{X}=$ B, N, O; Al, Si, P, S; Ga, Ge, As, Se; In, Sn, Sb, Te; Tl, Pb, Bi) using dispersion-corrected density functional theory method. The oxygen reduction activity of 14 catalysts was analysed in details, and the Gibbs free energy of each element reaction was calculated. According to the scaling relationship between $\Delta G_{\mathrm{OOH}^{*}}$ and $\Delta G_{\mathrm{OH}^{*}}$, the rate-determining steps of the remaining 20 catalysts were further analyzed. The results show that when the ORR reaction proceeds in the path $\mathrm{O}_{2} \rightarrow \mathrm{OOH}^{*} \rightarrow \mathrm{O}^{*} \rightarrow \mathrm{OH}^{*}$ $\rightarrow \mathrm{OH}^{-}$, the reaction energy barrier of Te-SV, Sb-DV, Pb-SV (unstable), Pb-DV (unstable), As-SV, As-DV, B-SV, N-SV and Sn$\mathrm{SV}$ is lower than $0.8 \mathrm{eV}$. Our result provides a theoretical basis for further exploration of carbon-based SACs or co-doped catalysts for ORR. 


\section{Conflicts of interest}

There are no conflicts to declare.

\section{Acknowledgements}

Supported by the "Transformational Technologies for Clean Energy and Demonstration", Strategic Priority Research Program of the Chinese Academy of Sciences, Grant No. XDA21090300 and National Natural Science Foundation of China, Grant No. 91834301. The DFT calculations utilized resources at the High Performance Computing Center, Jilin University.

\section{References}

1 Z. W. Chen, D. Higgins, A. P. Yu, L. Zhang and J. J. Zhang, A review on non-precious metal electrocatalysts for PEM fuel cells, Energy Environ. Sci., 2011, 4(9), 3167-3192.

2 M. Shao, Q. Chang, J. P. Dodelet and R. Chenitz, Recent Advances in Electrocatalysts for Oxygen Reduction Reaction, Chem. Rev., 2016, 116(6), 3594-3657.

3 Y. Nie, L. Li and Z. D. Wei, Recent advancements in Pt and Ptfree catalysts for oxygen reduction reaction, Chem. Soc. Rev., 2015, 44(8), 2168-2201.

4 Y. G. Li, W. Zhou, H. L. Wang, L. M. Xie, Y. Y. Liang, F. Wei, J. C. Idrobo, S. J. Pennycook and H. J. Dai, An oxygen reduction electrocatalyst based on carbon nanotubegraphene complexes, Nat. Nanotechnol., 2012, 7(6), 394-400.

5 H. B. Wang, T. Maiyalagan and X. Wang, Review on Recent Progress in Nitrogen-Doped Graphene: Synthesis, Characterization, and Its Potential Applications, ACS Catal., 2012, 2(5), 781-794.

6 L. T. Qu, Y. Liu, J. B. Baek and L. M. Dai, Nitrogen-Doped Graphene as Efficient Metal-Free Electrocatalyst for Oxygen Reduction in Fuel Cells, ACS Nano, 2010, 4(3), 1321-1326.

7 S. Y. Wang, L. P. Zhang, Z. H. Xia, A. Roy, D. W. Chang, J. B. Baek and L. M. Dai, BCN Graphene as Efficient MetalFree Electrocatalyst for the Oxygen Reduction Reaction, Angew. Chem., Int. Ed., 2012, 51(17), 4209-4212.

8 Y. Zheng, Y. Jiao, L. Ge, M. Jaroniec and S. Z. Qiao, Two-Step Boron and Nitrogen Doping in Graphene for Enhanced Synergistic Catalysis, Angew. Chem., Int. Ed., 2013, 52(11), 3110-3116.

9 C. H. Choi, S. H. Park and S. I. Woo, Binary and Ternary Doping of Nitrogen, Boron, and Phosphorus into Carbon for Enhancing Electrochemical Oxygen Reduction Activity, ACS Nano, 2012, 6(8), 7084-7091.

10 S. B. Yang, L. J. Zhi, K. Tang, X. L. Feng, J. Maier and K. Mullen, Efficient Synthesis of Heteroatom ( $\mathrm{N}$ or $\mathrm{S}$ )Doped Graphene Based on Ultrathin Graphene OxidePorous Silica Sheets for Oxygen Reduction Reactions, $A d v$. Funct. Mater., 2012, 22(17), 3634-3640.

11 J. T. Jin, F. P. Pan, L. H. Jiang, X. G. Fu, A. M. Liang, Z. Y. Wei, J. Y. Zhang and G. Q. Sun, Catalyst-Free Synthesis of Crumpled Boron and Nitrogen Co-Doped Graphite Layers with Tunable Bond Structure for Oxygen Reduction Reaction, ACS Nano, 2014, 8(4), 3313-3321.

12 K. Gong, F. Du, Z. Xia, M. Durstock and L. Dai, NitrogenDoped Carbon Nanotube Arrays with High Electrocatalytic Activity for Oxygen Reduction, Science, 2009, 323, 760-764.

13 Z. H. Sheng, H. L. Gao, W. J. Bao, F. B. Wang and X. H. Xia, Synthesis of boron doped graphene for oxygen reduction reaction in fuel cells, J. Mater. Chem, 2012, 22, 390-395.

14 Y. Zhao, L. Yang, S. Chen, X. Wang, Y. Ma, Q. Wu, Y. Jiang, W. Qian and Z. Hu, Can Boron and Nitrogen Co-doping Improve Oxygen Reduction Reaction Activity of Carbon Nanotubes?, J. Am. Chem. Soc., 2013, 135(4), 1201-1204.

15 Z. Yang, Z. Yao, G. Li, G. Fang, H. Nie, Z. Liu, X. Zhou, $X$. a. Chen and S. Huang, Sulfur-Doped Graphene as an Efficient Metal-free Cathode Catalyst for Oxygen Reduction, ACS Nano, 2012, 6, 205-211.

16 R. Li, Z. D. Wei, X. L. Gou and W. Xu, Phosphorus-doped graphene nanosheets as efficient metal-free oxygen reduction electrocatalysts, $R S C$ Adv., 2013, 3(25), 9978-9984.

17 X. Gong, S. S. Liu, C. Y. Ouyang, P. Strasser and R. Z. Yang, Nitrogen- and Phosphorus-Doped Biocarbon with Enhanced Electrocatalytic Activity for Oxygen Reduction, ACS Catal., 2015, 5(2), 920-927.

18 C. Chowdhury, S. Karmakar and A. Datta, Monolayer Group IV-VI Monochalcogenides: Low-Dimensional Materials for Photocatalytic Water Splitting, J. Phys. Chem. C, 2017, 121(14), 7615-7624.

19 S. Karmakar, C. Chowdhury and A. Datta, Noble-MetalSupported GeS Monolayer as Promising Single-Atom Catalyst for CO Oxidation, J. Phys. Chem. C, 2018, 122(26), 14488-14498.

20 C. Chowdhury and A. Datta, Silicon-Doped NitrogenCoordinated Graphene as Electrocatalyst for Oxygen Reduction Reaction, J. Phys. Chem. C, 2018, 122(48), 27233-27240.

21 C. Chowdhury and A. Datta, Doped boron nitride surfaces: potential metal free bifunctional catalysts for non-aqueous Li-O-2 batteries, Phys. Chem. Chem. Phys., 2018, 20(24), 16485-16492.

22 N. Larouche, R. Chenitz, M. Lefevre, E. Proietti and J. P. Dodelet, Activity and stability in proton exchange membrane fuel cells of iron-based cathode catalysts synthesized with addition of carbon fibers, Electrochim. Acta, 2014, 115, 170-182.

23 G. Wu, K. L. More, C. M. Johnston and P. Zelenay, HighPerformance Electrocatalysts for Oxygen Reduction Derived from Polyaniline, Iron, and Cobalt, Science, 2011, 332(6028), 443-447.

24 L. Yu, X. L. Pan, X. M. Cao, P. Hu and X. H. Bao, Oxygen reduction reaction mechanism on nitrogen-doped graphene: a density functional theory study, J. Catal., 2011, 282(1), 183-190.

25 X. M. Zhang, S. S. Yu, H. Chen and W. T. Zheng, TM atoms on $\mathrm{B} / \mathrm{N}$ doped defective graphene as a catalyst for oxygen reduction reaction: a theoretical study, $R S C A d v ., 2015$, 5(101), 82804-82812. 
26 X. Zhang, S. Shi, T. Gu, L. Li and S. Yu, The catalytic activity and mechanism of oxygen reduction reaction on P-doped Mos2, Phys. Chem. Chem. Phys., 2018, 20(27), 18184-18191.

27 N. Yang, L. Li, J. Li, W. Ding and Z. D. Wei, Modulating the oxygen reduction activity of heteroatom-doped carbon catalysts via the triple effect: charge, spin density and ligand effect, Chem. Sci., 2018, 9(26), 5795-5804.

28 J. K. Nørskov, J. Rossmeisl, A. Logadottir, L. Lindqvist, J. R. Kitchin and T. Bligaard, Origin of the Overpotential for Oxygen Reduction at a Fuel-Cell Cathode, J. Phys. Chem. $B, 2004,108,17886$.

29 I. C. Man, H. Y. Su, F. Calle-Vallejo, H. A. Hansen, J. I. Martinez, N. G. Inoglu, J. Kitchin, T. F. Jaramillo, J. K. Norskov and J. Rossmeisl, Universality in Oxygen Evolution Electrocatalysis on Oxide Surfaces, Chemcatchem, 2011, 3(7), 1159-1165.

30 B. Delley, From molecules to solids with the $\operatorname{DMol}(3)$ approach, J. Chem. Phys., 2000, 113(18), 7756-7764.

$31 \mathrm{~S}$. Grimme, Semiempirical GGA-type density functional constructed with a long-range dispersion correction, $J$. Comput. Chem., 2006, 27(15), 1787-1799.

32 J. P. Perdew, K. Burke and M. Ernzerhof, Generalized Gradient Approximation Made Simple, Phys. Rev. Lett., 1996, 77, 3865-3868.

33 B. Delley, An all-electron numerical method for solving the local density functional for polyatomic molecules, J. Chem. Phys., 1990, 92, 508-517.

34 H. J. Monkhorst and J. D. Pack, Special Points for BrillouinZone Integrations, Phys. Rev. B, 1976, 13(12), 5188-5192.

35 A. Klamt and G. Schuurmann, Cosmo - a New Approach to Dielectric Screening in Solvents with Explicit Expressions for the Screening Energy and Its Gradient, J. Chem. Soc., Perkin Trans. 2, 1993, (5), 799-805.

36 D. E. Jiang, B. G. Sumpter and S. Dai, Unique chemical reactivity of a graphene nanoribbon's zigzag edge, J. Chem. Phys., 2007, 126(13), 134701.

37 L. Zhang, J. Niu, M. Li and Z. Xia, Catalytic Mechanisms of Sulfur-Doped Graphene as Efficient Oxygen Reduction Reaction Catalysts for Fuel Cells, J. Phys. Chem. C, 2014, 118(7), 3545-3553.

38 M. T. Li, L. P. Zhang, Q. Xu, J. B. Niu and Z. H. Xia, N-doped graphene as catalysts for oxygen reduction and oxygen evolution reactions: Theoretical considerations, J. Catal., 2014, 314, 66-72.

39 M. Kaukonen, A. V. Krasheninnikov, E. Kauppinen and R. M. Nieminen, Doped Graphene as a Material for Oxygen Reduction Reaction in Hydrogen Fuel Cells: A Computational Study, ACS Catal., 2013, 3(2), 159-165.

40 X. Zhang, Z. Lu, Z. Fu, Y. Tang, D. Ma and Z. Yang, The mechanisms of oxygen reduction reaction on phosphorus doped graphene: a first-principles study, J. Power Sources, 2015, 276, 222-229.

$41 \mathrm{H}$. Wang and W. An, Promoting the oxygen reduction reaction with gold at step/edge sites of Ni@AuPt core-shell nanoparticles, Catal. Sci. Technol., 2017, 7(3), 596-606.

42 H. Wang, W. An, X. Y. Liu and C. H. Turner, Oxygen reduction reaction on $\mathrm{Pt}(111), \mathrm{Pt}(221)$, and $\mathrm{Ni} / \mathrm{Au} 1 \mathrm{Pt} 3(221)$ surfaces: probing scaling relationships of reaction energetics and interfacial composition, Chem. Eng. Sci., 2018, 184, 239-250. 